\title{
Globe
}

Revue internationale d'études québécoises

\section{Chronologie des études québécoises}

Volume 4, numéro 2, 2001

Les études québécoises dans le monde

URI : https://id.erudit.org/iderudit/1000633ar

DOI : https://doi.org/10.7202/1000633ar

Aller au sommaire du numéro

Éditeur(s)

Globe, Revue internationale d'études québécoises

ISSN

1481-5869 (imprimé)

1923-8231 (numérique)

Découvrir la revue

Citer ce document

(2001). Chronologie des études québécoises. Globe, 4(2), 49-57.

https://doi.org/10.7202/1000633ar d'utilisation que vous pouvez consulter en ligne.

https://apropos.erudit.org/fr/usagers/politique-dutilisation/ 


\section{Chronologie des études québécoises}

\section{8}

Création du Centre de recherche en littérature canadienne-française, à l'Université d'Ottawa, par Paul Wyczynski

Création du School of Canadian Studies, à l'Université Carleton, à Ottawa, en Ontario

Création du Canadian Studies Centre à la Michigan State University, à East Lansing, aux États-Unis

\section{2}

Publication de Situation de la recherche sur le Canada français, sous la direction de Fernand Dumont et Yves Martin.

\section{3}

Création du Centre d'études canadiennes-françaises à l'Université McGill, par Michael Olivier

Création du Centre de littérature canadienne-française à l'Université de Montréal, par Réginald Hamel

Création du John F. Kennedy-Institut für Nordamerika-studien à la Freie Universität Berlin, en Allemagne

\section{4}

Création du Canadian Studies Program, à l'University of Vermont, à Burlington, aux États-Unis

\section{6}

Fondation de la Revue d'études canadiennes

Création du Center for the Study of Canada, à la State University of New York at Plattsburgh, État de New York, aux États-Unis

Daniel Chartier, "Chronologie des études québécoises", Globe. Revue internationale d'études québécoises, vol. 4, nº 2, 2001. 


\section{REVUE INTERNATIONALE D'ÉTUDES QUÉBÉCOISES}

1967

Fondation de la revue Voix et images du pays

Création du Canadian-American Center, à l'University of Maine, à Orono, aux États-Unis

\section{8}

Le Centre de recherche en littérature canadienne-française de l'Université d'Ottawa devient le Centre de recherche en civilisation canadienne-française (CRCCF)

Création d'une Chaire en littérature canadienne-française à l'Université de Rennes, en France

Création du Centre d'études acadiennes, à l'Université de Moncton, au Nouveau-Brunswick

Création de L'Institut Indo-Canadien Shastri, en Inde

Création du Canadian Studies Centre, à l'Université d'Umeå, en Suède

\section{9}

Le Centre de littérature canadienne-française de l'Université de Montréal est démantelé

\section{1}

Fondation de l'Association for Canadian Studies in the United States (ACSUS)

\section{2}

Création de l'Institute of the USA and Canada à l'Académie des Sciences d'Union soviétique, à Moscou

\section{3}

Fondation de The American Review of Canadian Studies

Fondation de l'Association d'études canadiennes, à l'Université Queen's de Kingston, en Ontario

\section{4}

Création du Centre d'études canadiennes de Bordeaux, à la Maison des

Sciences de l'Homme d'Aquitaine, à Talence, en France 


\section{CHRONOLOGIE DES ÉTUDES QUÉBÉCOISES}

\section{5}

Le Centre de littérature canadienne-française de l'Université de Montréal renaît sous le nom de Centre d'études québécoises (CÉTUQ)

La revue Voix et images du pays devient Voix et images

Fondation de la British Association for Canadian Studies (BACS)

Fondation de la revue Études canadiennes, Canadian Studies : revue interdisciplinaire des études canadiennes en France

Création du Centre of Canadian Studies à l'Université d'Édimbourg, en Écosse

\section{6}

Fondation de l'Association française d'études canadiennes, à Paris

Création du Centre d'études québécoises de l'Université de Liège, en Belgique

Création du Centre d'études canadiennes, à l'Université de Grenoble, en France

Creation de l'American-Canadian Studies Institute, à l'Université nationale Chungnam, à Taejon, en Corée du Sud

\section{7}

Fondation de l'Association japonaise d'études canadiennes

\section{8}

Création d'un Centre d'études québécoises à l'Université de Trèves, en Allemagne

Création du Centre d'études franco-canadiennes de l'Ouest, au Collège universitaire de Saint-Boniface, au Manitoba

\section{9}

Premier Quebec Summer Seminar à la State University of New York at Plattsburgh, aux États-Unis

Fondation de l'Association italienne d'études canadiennes

Fondation de la revue The Annual Review of Canadian Studies, à Tokyo, au Japon

Création de l'Institut québécois de recherche sur la culture, par Fernand Dumont 


\section{REVUE INTERNATIONALE D'ÉTUDES QUÉBÉCOISES}

1980

Parution d'un l'article de Pierre Savard, "Études canadiennes et québécoises : esquisse de bilan et réflexion"

Fondation de la Gesellschaft für Kanada-Studien, à Gummersbach, en Allemagne

Fondation du Northeast Council for Quebec Studies, à Radford, aux États-Unis

Création du Canadian Studies Centre, à l'Aarhus Universitet, au Danemark

\section{1}

Fondation de la revue Zeitschrift der gesellschaft für Kanada-Studien

Création du Conseil international d'études canadiennes (CIÉC)

Création du Centre de recherche en littérature québécoise (CRELIQ), à l'Université Laval

Création d'un Centre d'études canadiennes, à l'Université Libre de Bruxelles

\section{2}

Fondation de l'Association d'études canadiennes en Australie et en Nouvelle-Zélande

Fondation de l'Association irlandaise d'études canadiennes, à Galway

Création du Centre for Canadian Studies, à l'Université Maharaja Sayajirao de Baroda, en Inde

Création du Centre d'études franco-canadiennes, à l'Université de Varsovie

Création du Canadian Studies Centre, à l'Université d'études internationales de Séchuan, à Chongqing, en Chine

\section{3}

Publication d'un numéro spécial d'Études littéraires : " Regards du Brésil sur le Québec "

Fondation de la revue Australian-Canadian Studies Fondation de la revue Québec Studies, aux États-Unis

Création du Centre for North American Studies, à la Lunds Universitet, en Suède 


\section{CHRONOLOGIE DES ÉTUDES QUÉBÉCOISES}

\section{4}

Le Northeast Council for Quebec Studies devient l'American Council for Quebec Studies

Fondation de The London Journal of Canadian Studies

Fondation de l'Association nordique d'études canadiennes, à Aarhus au Danemark

Fondation de l'Association chinoise d'études canadiennes, à Chongqing Création du Centre de coopération inter-universitaire franco-québécoise, à Paris

Création du Programme d'études canadiennes par le gouvernement fédéral

Création du Centro di Studi Quebecchesi, à l'Université de Bologne, en Italie

Création du Centre for Canadian Studies, à l'Université Yonsei de Séoul, en Corée du Sud

Création du Centre Jacques-Cartier, à l'Université Lumière-Lyon II, en France

\section{5}

Fondation de la Studiegenootschap Canada, à La Haye, aux Pays-Bas

Fondation de l'Association indienne d'études canadiennes, à l'Université Maharaja Sayajirao de Baroda

Fondation de l'Association des études canadiennes en Israël, à Jérusalem Création d'un Centre d'études québécoises à l'Université des études internationales de Shanghai, en Chine

Création de l'Institut für Kanada-studien, à l'Université d'Augsburg, en Allemagne

Création du Centre of Canadian Studies à la Queen's University of Belfast, en Irlande du Nord

1987

Création du Centre Saint-Laurent à l'Institut d'études politiques, à Aixen-Provence, en France 
REVUE INTERNATIONALE D'ÉTUDES QUÉBÉCOISES

\section{8}

Fondation de l'Associación Española de Estudios Canadienses, à Madrid Fondation de la Gesellschaft zur Förderung von Nordamerika-studien an der Universität Wien, en Autriche

Création du Centro Cultural Canadá-Córdoba-Argentina, à l'Universidad Nacional de Córdoba, en Argentine

Création du Centre d'études canadiennes, à la Rijksuniversiteit Groningen, aux Pays-Bas

Création du Centre d'études québécoises, à l'Odense Universitet, au Danemark

Création de I'Institute of American and Canadian Studies, à l'Université Sophia, à Tokyo, au Japon, issu du Centre d'études canadiennes fondé par Conrad Fortin auparavant

\section{9}

Publication de La littérature québécoise à l'étranger. Guide aux usagers, par André Vanasse

Fondation de la revue Canada Yon-Ku, à l'Université Yonsei de Séoul, en Corée du Sud

Fondation de l'Association soviétique d'études canadiennes, à Moscou

Création du Centre de recherches sur la Francophonie, à l'Université nationale de Séoul, en Corée du Sud

1990

Parution de Plus ou moins. The State of Québec Studies in the United States, 1990, par Robert M. Gill et Jeanne Kissner

Fondation de la Revista Española de Estudios Canadienses, à Madrid

Fondation de la revue Études canadiennes, en Chine

Fondation de la Revue d'études canadiennes, en Italie

Création du Centre for Quebec Studies, à l'University of New England, en Australie

Création du Centre d'études sino-canadiennes et pour la coopération académique avec le Canada, à l'Université de Beijing (Beida), en Chine

Création du Centre for Canadian Studies à la Karlova Univerzita, à Prague, en République tchèque 


\section{CHRONOLOGIE DES ÉTUDES QUÉBÉCOISES}

\section{1}

Fondation de l'Association vénézuélienne d'études canadiennes

Création du Núcleo de Estudos Canadenses, à l'Universidade Federal Fluminense, à Niterói, au Brésil

\section{2}

Fondation de l'Association mexicaine d'études canadiennes

Création du Centre d'études canadiennes et québécoises à l'Université Babes-Bolyai, à Cluj-Napoca, en Roumanie

Création de l'Institut d'études acadiennes et québécoises, à l'Université de Poitiers, en France

Création du Canadian and Québec Studies Programme, à l'Université Eötvös Loránd, à Budapest, en Hongrie

Création du Halbert Centre for Canadian Studies à l'Université hébraïque de Jérusalem, en Israël

Création du Núcleo de Estudos Canadenses, à l'Universidade Federal do Rio Grande do Sul, à Porto Alegre, au Brésil

\section{3}

Colloque "Les études québécoises en Europe ", organisé par Jean-Marie Klinkenberg

Le Centre d'études canadiennes-françaises de l'Université McGill devient le Programme d'études sur le Québec (PÉQ)

Parution du Répertoire des études littéraires québécoises en Europe, de Gilles Dorion

Parution de Northern Exposures. Scholarship on Canada in the United States, sous la direction de Karen Gould, Joseph T. Jockel et William Metcalfe

Fondation du Centre interuniversitaire d'études québécoises (CIÉQ) à l'Université Laval et à l'Université du Québec à Trois-Rivières

Création du Centro de Estudios Canadienses de Cáceres, à l'Universidad de Extremadura, en Espagne

Création du Baltic Center for North American Studies, à l'Université de Tartu, en Estonie

Création du Canadian Studies Centre, à l'Univerzita Komensheko, à Bratislava, en Slovaquie 


\section{4}

L'Institut québécois de recherche sur la culture (IQRC) devient le Centre Culture et Société de l'Institut national de la recherche scientifique, une constituante de l'Université du Québec

Création du Centre d'étude de la chanson québécoise, à la Universität Innsbruck, en Autriche

Création du Centro de Estudios Canadienses, à l'Universidad Central de Venezuela, à Caracas

Création de la Cátedra de Estudios sobre Canadá, à l'Université de La Havane, à Cuba

\section{6}

Parution de Canadian Studies in Italy. Études canadiennes en Italie, par Caterina Ricciardi

Parution de Le point sur les études canadiennes. Les années 90, par David Cameron

\section{7}

Fondation de l'Association internationale des études québécoises (AIÉQ)

Création du Centre d'études québécoises, à l'Université de Leicester, au Royaume-Uni

Création du Centre d'études francophones Québec-Pacifique, à l'Université Simon Fraser, en Colombie-Britannique

Création du Centre Moscou-Québec, à l'Université d'État des sciences humaines de Russie

\section{8}

Fondation de Globe, revue internationale d'études québécoises

Création du Centre interuniversitaire d'études québécoises, aux Universités de Bologne, Turin, Bari et Urbino

\section{9}

Création d'un Centre d'études canadiennes à l'Université de Graz, en Autriche 


\section{CHRONOLOGIE DES ÉTUDES QUÉBÉCOISES}

2000

Fermeture du Centre d'études québécoises, à l'Université de Trèves, en Allemagne

Création du Paraguayan Centre for Canadian Studies, à l'Universidad Evangelica del Paraguay, à Asuncion

\section{1}

Colloque "L'émergence des études québécoises dans le monde ", à Sherbrooke

Parution de l'État des lieux de la recherche sur le Canada en France (1976-2001), sous la direction de Jean-Michel Lacroix

Fondation de la revue Interfaces Brasil-Canada

Création d'une Chaire en études québécoises à l'Université de Leeds, attribuée à Rachel Killick

Création d'une Chaire d'études québécoises, à l'Université York, attribuée à Jean-Louis Roy 